\title{
Desertification mapping based ARIMA model and Landsat time series
}

\author{
Raul Toma ${ }^{1}$, Thiago Santos ${ }^{2}$, Adunias Teixeira ${ }^{2}$, Fabrizio Terra $^{3}$, and Luis Clenio Moreira ${ }^{4}$ \\ ${ }^{1}$ Universidade Federal do Ceará \\ ${ }^{2}$ Universidade Federal do Ceara \\ ${ }^{3}$ Federal University of Jequitinhonha and Mucuri Valleys (UFVJM) \\ ${ }^{4}$ Instituto Federal de Educacao Ciencia e Tecnologia do Ceara
}

May 5, 2020

\begin{abstract}
Desertification models do not include analyses of rainfall, temporality, field visits and projection scenarios. The objective is to evaluate desertification under levels of vegetation, soil and albedo, construct forecast models and analyze its variability with time and rainfall. Landsat TM was used for NDVI, TGSI and albedo between 2000 and 2008 in dry and rainy periods in Irauçuba Centro Norte (ICN) and in the National Forest of Sobral (FLONA). Desertification levels and indices of 30 pixels were quantified and their means were inputs in the ARIMA model. The results showed vegetation and albedo at different levels of desertification. The medium desertification class of NDVI increased by $18 \%$ at ICN and the low desertification class of albedo increased by $3 \%$. TGSI showed the class severe at ICN and FLONA. The AR(3) model indicated reduction in NDVI, while MA(1) for TGSI and albedo showed severe and low level. ARMA $(2,1)$ showed optimistic scenario of recovery for pixels with desertified NDVI and Albedo of FLONA. The AR(3) model for TGSI of FLONA showed a reduction of 0.0006504 in 70 years. Anthropic practices and climate were responsible for the desertification of ICN, while FLONA was shown to be non-desertified, but NDVI and TGSI were affected by the anthropic practices. Therefore, desertification showed different levels at ICN, rainfall and time affected vegetation and albedo at ICN and FLONA. ARMA $(2,1)$ models showed that the prospects of desertification reversal are greater for FLONA.
\end{abstract}

\section{Hosted file}

Manuscript_Desertification mapping based ARIMA model and Landsat time series.docx available at https://authorea.com/users/299190/articles/428782-desertification-mapping-based-arimamodel-and-landsat-time-series 

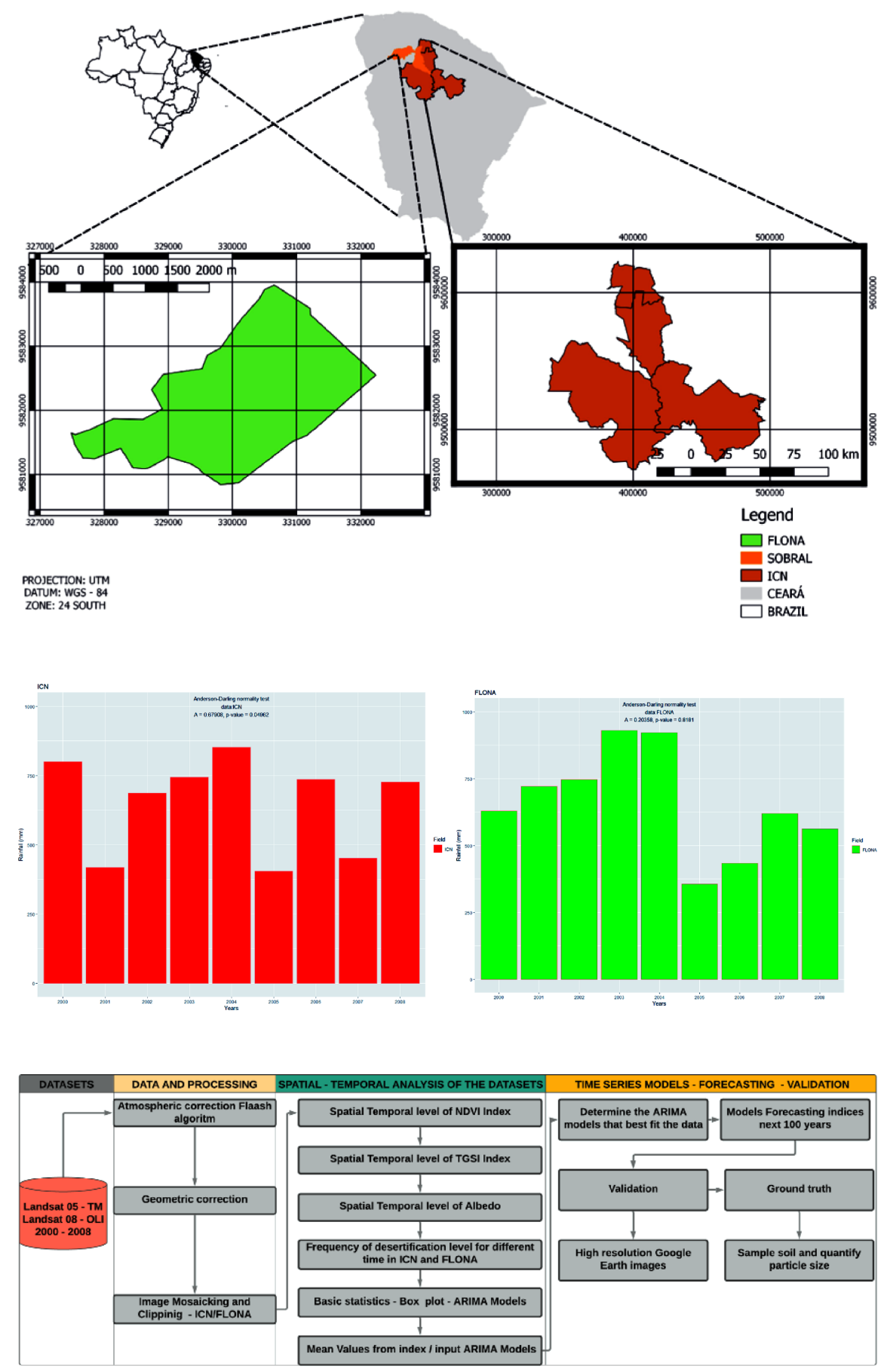

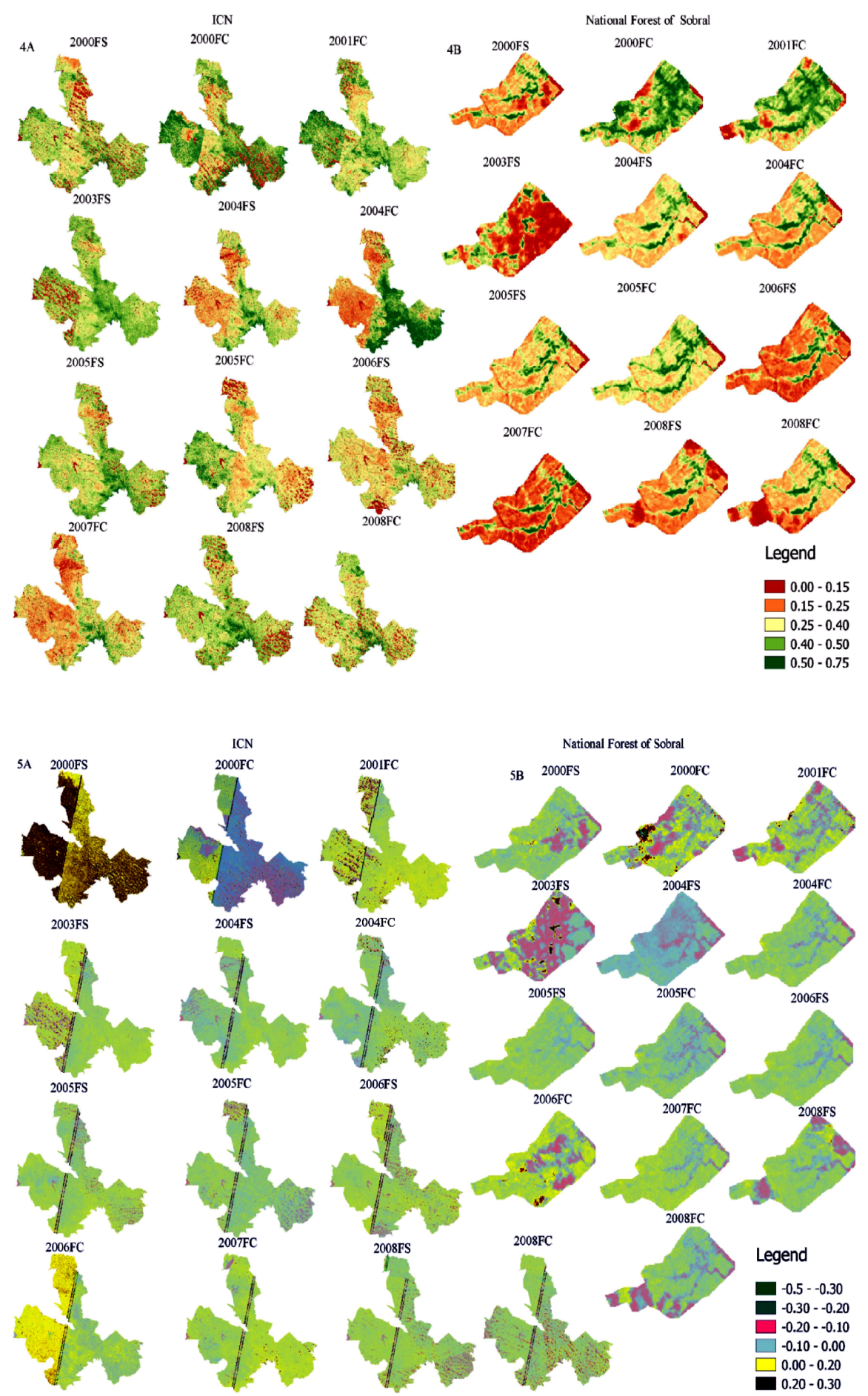

Legend

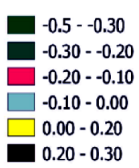



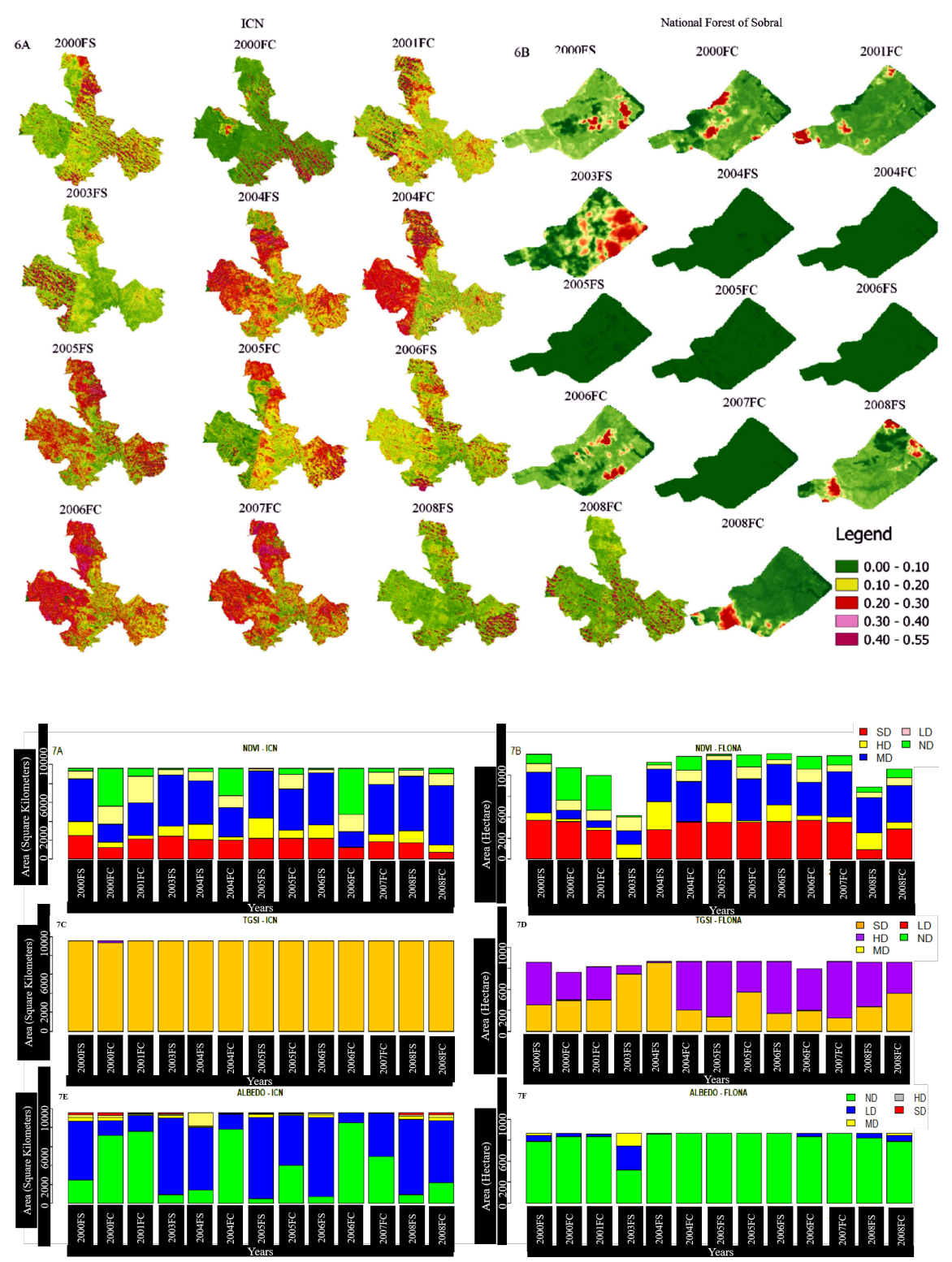

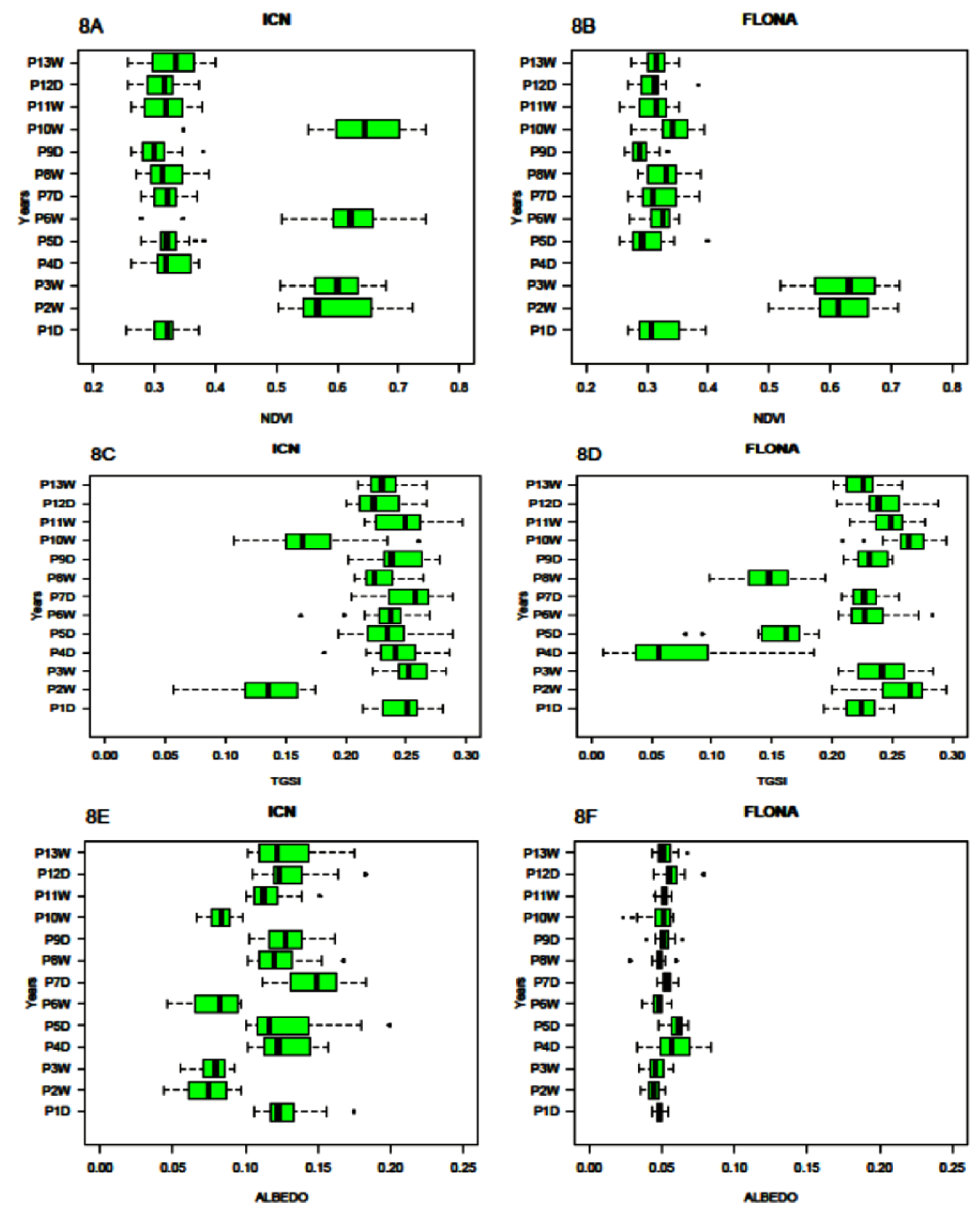

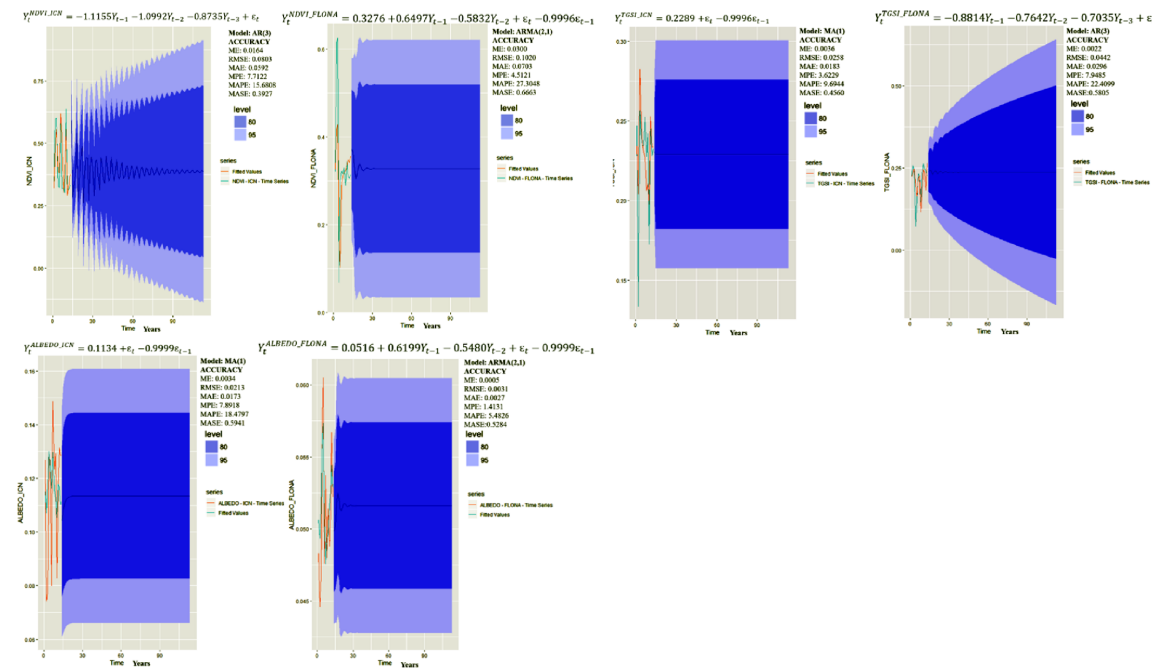